\title{
Continuous mapping of the corticospinal tracts in intramedullary spinal cord tumor surgery using an electrified ultrasonic aspirator
}

\author{
Ori Barzilai, MD, ${ }^{1}$ Zvi Lidar, MD, ${ }^{1}$ Shlomi Constantini, MD, MSc, ${ }^{1,2}$ Khalil Salame, MD, ${ }^{1}$ \\ Yifat Bitan-Talmor, MMedSc, ${ }^{1}$ and Akiva Korn, MMedSc, D-ABNM ${ }^{1}$ \\ 'Department of Neurosurgery, Tel Aviv, "Sourasky" Medical Center, Tel Aviv University; and 2Department of Pediatric \\ Neurosurgery, "Dana" Children's Hospital, Tel Aviv Medical Center, Tel Aviv, Israel
}

Intramedullary spinal cord tumors (IMSCTs) represent a rare entity, accounting for $4 \%-10 \%$ of all central nervous system tumors. Microsurgical resection of IMSCTs is currently considered the primary treatment modality. Intraoperative neurophysiological monitoring (IONM) has been shown to aid in maximizing tumor resection and minimizing neurological morbidity, consequently improving patient outcome. The gold standard for IONM to date is multimodality monitoring, consisting of both somatosensory evoked potentials, as well as muscle-based transcranial electric motor evoked potentials (tcMEPs). Monitoring of tcMEPs is optimal when combining transcranial electrically stimulated muscle tcMEPs with D-wave monitoring. Despite continuous monitoring of these modalities, when classic monitoring techniques are used, there can be an inherent delay in time between actual structural or vascular-based injury to the corticospinal tracts (CSTs) and its revelation. Often, tcMEP stimulation is precluded by the surgeon's preference that the patient not twitch, especially at the most crucial times during resection. In addition, D-wave monitoring may require a few seconds of averaging until updating, and can be somewhat indiscriminate to laterality. Therefore, a method that will provide immediate information regarding the vulnerability of the CSTs is still needed.

The authors performed a retrospective series review of resection of IMSCTs using the tip of an ultrasonic aspirator for continuous proximity mapping of the motor fibers within the spinal cord, along with classic muscle-based tcMEP and D-wave monitoring.

The authors present their preliminary experience with 6 patients who underwent resection of an IMSCT using the tip of an ultrasonic aspirator for continuous proximity mapping of the motor fibers within the spinal cord, together with classic muscle-based tcMEP and D-wave monitoring. This fusion of technologies can potentially assist in optimizing resection while preserving neurological function in these challenging surgeries.

https://thejns.org/doi/abs/10.3171/2016.12.SPINE16985

KEY WORDS IMSCT; intramedullary spinal cord tumor; spine surgery; intraoperative nerve monitoring; mapping; ultrasonic aspirator; diagnostic technique

I NTRAMEDULLARY spinal cord tumors (IMSCTs) represent a rare entity in children and adults. ${ }^{26,33}$ The most common intramedullary tumors can be classified as primary neoplasms or, less commonly, metastatic lesions. ${ }^{19}$ Ependymomas are common in adults, while astrocytomas are far more common in children. ${ }^{10}$ Most ependymomas have relatively demarcated borders, while astrocytomas are more infiltrative and need to be resected until a white matter "interphase" appears. ${ }^{20}$

Microsurgical resection of IMSCTs is currently considered the primary treatment modality, while radiotherapy and/or chemotherapy are reserved for recurrent or malignant tumors..$^{3,9,41}$ The observation that the majority of IMSCTs are benign and consequently gross-total removal might result in long-term survival further supports the need for safe resection. ${ }^{10}$ The extent of resection has been correlated with progression-free survival ${ }^{4}$ and lower recurrence rates. ${ }^{21}$ Advances in microsurgery have contributed to safer resection ability. However, despite all advances, surgery for IMSCTs is still very challenging and may carry significant morbidity. Proximity to crucial neural elements necessitates use of surgical adjuncts such as high-

ABBREVIATIONS CMAP = compound motor action potential; CST = corticospinal tract; IMSCT = intramedullary spinal cord tumor; IONM = intraoperative neurophysiological monitoring; SSEP = somatosensory evoked potential; tcMEP = transcranial electric motor evoked potential.

SUBMITTED August 22, 2016. ACCEPTED December 30, 2016.

INCLUDE WHEN CITING Published online May 19, 2017; DOI: 10.3171/2016.12.SPINE16985. 
resolution MRI, intraoperative neurophysiological monitoring (IONM) of nerves, and intraoperative ultrasound. ${ }^{19}$ To date, the use of an ultrasonic aspirator as the resective tool during IMSCT surgery is considered state of the art. ${ }^{8}$

Intraoperative neurophysiological monitoring has been shown to play an important role in IMSCT surgery. ${ }^{6,34,35}$ Current common modalities enable continuous somatosensory evoked potential (SSEP) monitoring as well as motor potential monitoring, by both muscle-based transcranial electric motor evoked potentials (tcMEPs) and by D-wave monitoring. ${ }^{22,34}$ TcMEP monitoring alarms alert the surgeon when the corticospinal motor conduction has been compromised in both a reversible and nonreversible manner. It has previously been shown that loss of the muscle tcMEP signal predicts postoperative functional outcome ${ }^{28}$ as it corresponds to at least a short-term motor deficit postoperatively, ${ }^{23,28}$ while attenuation of over $50 \%$ of the D-wave amplitude correlates with long-term permanent motor injury. ${ }^{11}$ This allows for a tailored approach to surgery: maximizing resection while minimizing permanent neurological deficit.

The desire for continuous mapping of functional areas during resection with the ultrasonic aspirator, aiming at improving surgical strategy and maximizing resection, while preserving neural elements in spinal cord surgery, led to our current trial. Herein we present our preliminary experience with a multipurpose resective tool (i.e., ultrasonic aspirator connected to IONM) to allow real-time continuous mapping of the corticospinal tract (CST).

\section{Methods}

This study is in compliance with and was approved by the "Sourasky" Medical Center IRB.

\section{Resection}

The technique for resection of IMSCTs has been described extensively elsewhere. 7 An ultrasonic surgical aspirator (CUSA, Integra Life Sciences) was used for tumor resection in all 6 cases. The aspirator was introduced into the surgical field after entering the spinal cord, either through the midline or (in a single case) through the dorsal root entry zone. Our common practice in resection of intramedullary tumors is to begin with combined sharp and blunt separation of dorsal tumor margins, only when they are easily recognizable. When those margins become blurry to a "white matter interface," we almost always use an ultrasonic aspirator, at low amplitudes, for tumor resection up to the margins.

\section{CUSA Stimulation and Recording}

Spinal stimulation was applied using the CUSA 36$\mathrm{kHz}$ Hand Piece CEM Nosecone (Integra) (Fig. 1). All neurophysiological data were recorded using a 16/32 channel intraoperative evoked response unit (NIM Eclipse, Medtronic). Disposable subdermal needle and scalp electrodes were manufactured by Rhythmlink, and D-wave electrodes were manufactured by Ad Tech Medical Instrument Corp. Muscle-tcMEPs, D-waves, and SSEPs were monitored in a fashion described in our previous study, ${ }^{22}$ and the CUSA-mediated stimulation parameter settings

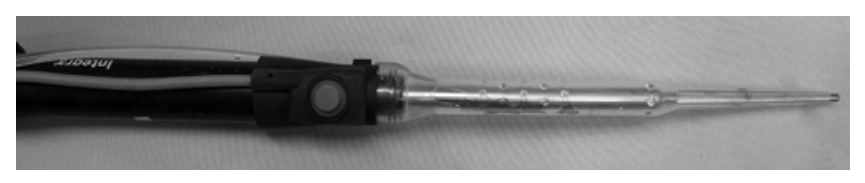

FIG. 1. The standard ultrasonic resective tool with the attached nosecone for an electrified tip.

were as follows: stimulation train-of-three pulses with an interstimulus interval of $3 \mathrm{msec}$, pulse duration $200 \mu \mathrm{sec}$, 1.2 trains per second, intensity range $0.5-2 \mathrm{~mA}$, in a monopolar cathodal fashion with the return electrode (anode) placed in the sterile field close to the wound.

Time-locked sweeps of $100 \mathrm{msec}$ were collected after each CUSA stimulation and analyzed for evoked compound motor action potential (CMAP) activity in the following muscles: for cervical-level tumors, the deltoid, biceps, triceps, thenar, and tibialis anterior muscles; for thoracic-level tumors, the quadriceps, tibialis anterior, and abductor hallucis muscles; and for lumbar-level tumors, the quadriceps, tibialis anterior, gastrocnemius, and abductor hallucis muscles (Table 1 lists the individual case settings).

All evoked activity detected was immediately brought to the surgeon's attention.

\section{Monitoring Intermodality Dynamics}

The standard protocol for cord stimulation was as follows: SSEP, tcMEP, and D-wave data were collected as the main monitoring modalities. After induction of anesthesia and positioning, SSEP and tcMEP baseline data were collected. SSEP data were averaged until an acceptable signal-to-noise level was reached, typically after 200-500 sweeps at $4.37 \mathrm{~Hz}$. High-amplitude artifacts, beyond 50 $\mathrm{mV}$, were automatically rejected. The time to final average, and thus updates to the surgeon, was about 1-2 minutes. Transcranial MEP data were collected separately in a single-train stimulus manner, with no averaging and therefore no "lag" time. When working within the cord or other sensitive areas in the wound, transcranial stimulation was performed in coordination with the surgeon to avoid injury emanating from the patient twitching. D-wave data were collected beginning at $\mathrm{D}$-wave electrode introduction, after dural opening. When necessary, averages of 5-10 sweeps were collected, at a stimulation repetition rate of $2 \mathrm{~Hz}$, leading to a maximum lag time of approximately 5 seconds. These modalities were collected in series, not in parallel.

At the point the CUSA was introduced into the surgical wound for tumor emulsification, the recording of muscle tcMEPs, SSEPs, and D-waves was halted. On the IONM unit, a separate physical stimulation port was used for CUSA stimulation, and a separate set of CMAP traces was collected and displayed on the IONM unit for this modality.

CUSA-based stimulation was undertaken in between D-wave and muscle tcMEP collection sets. If a duration of 30 seconds was noted without D-wave or muscle tcMEP updates, CUSA stimulation was halted in coordination with the surgeon, and these modalities were tested. Likewise, these modalities were tested each time an active 


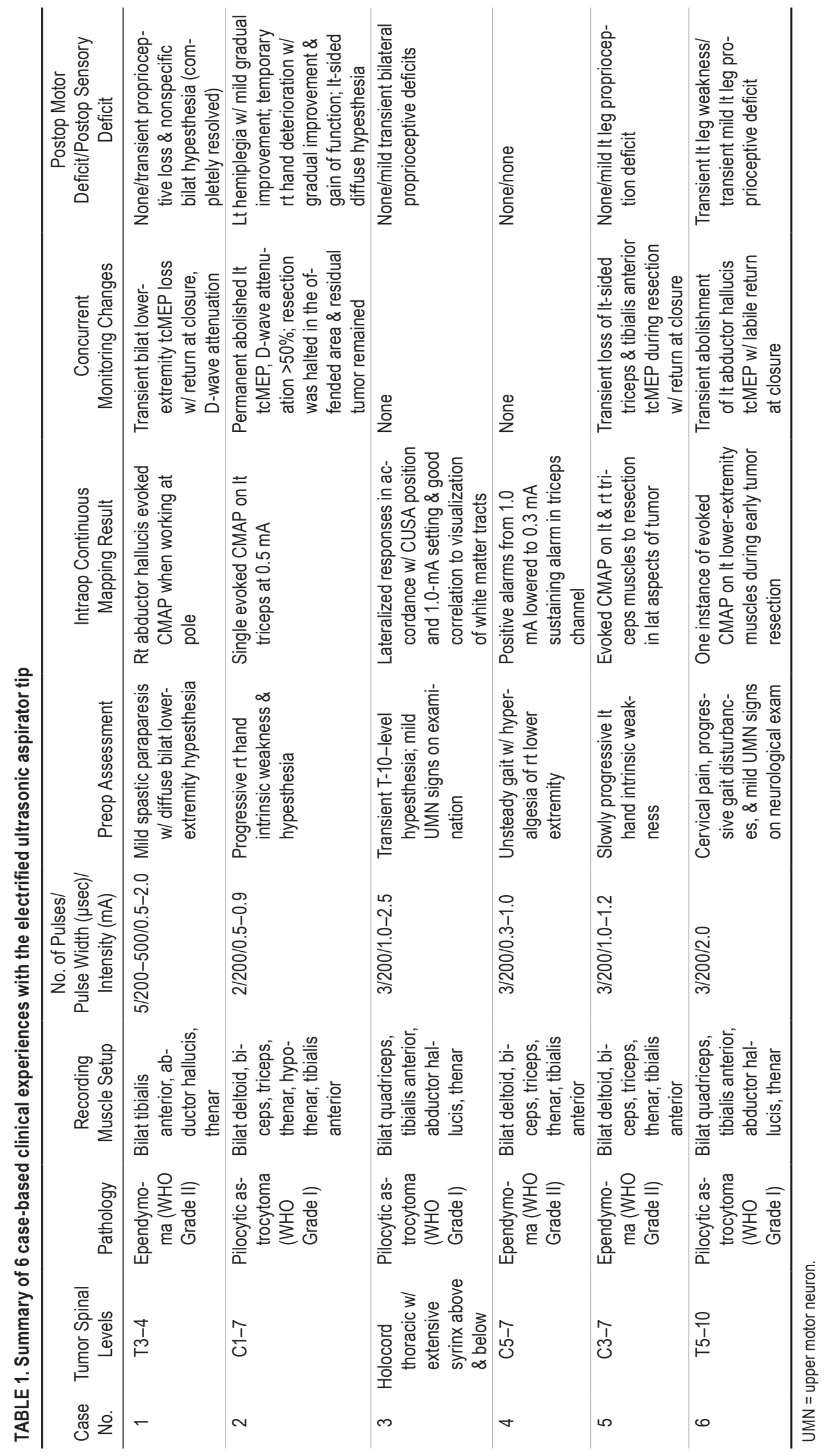


CUSA mapping alarm was raised. CUSA stimulation was independent of active CUSA emulsification as long as the CUSA tip remained within the spinal cord and closed the electrical circuit. No halting of normal CUSA function was necessary.

Significant attenuation or lability of the tcMEP or SSEP data was immediately brought to the attention of the surgical team for appropriate intervention, including pausing the procedure, shifting the resection focus, raising the patient's temperature, raising the patient's blood pressure, or irrigating with warm saline.

\section{Illustrative Case}

A previously healthy 36 -year-old man presented with 4 years of discomfort in his neck and upper back. The patient gradually developed diffuse sensory disturbances, more notably in both legs than in the arms. Several weeks prior to surgery, he developed mild spastic paraparesis and gait disturbances. MRI (Fig. 2) revealed a T3-4 contrastenhancing intramedullary lesion with both rostral and caudal syringes.

SSEP data were abolished at myelotomy. Left- and right-sided tcMEP data displayed lability and loss of data during deep stages of resection, with full recovery by closure. D-wave data were available both rostral and caudal to the tumor, with transient attenuation of approximately
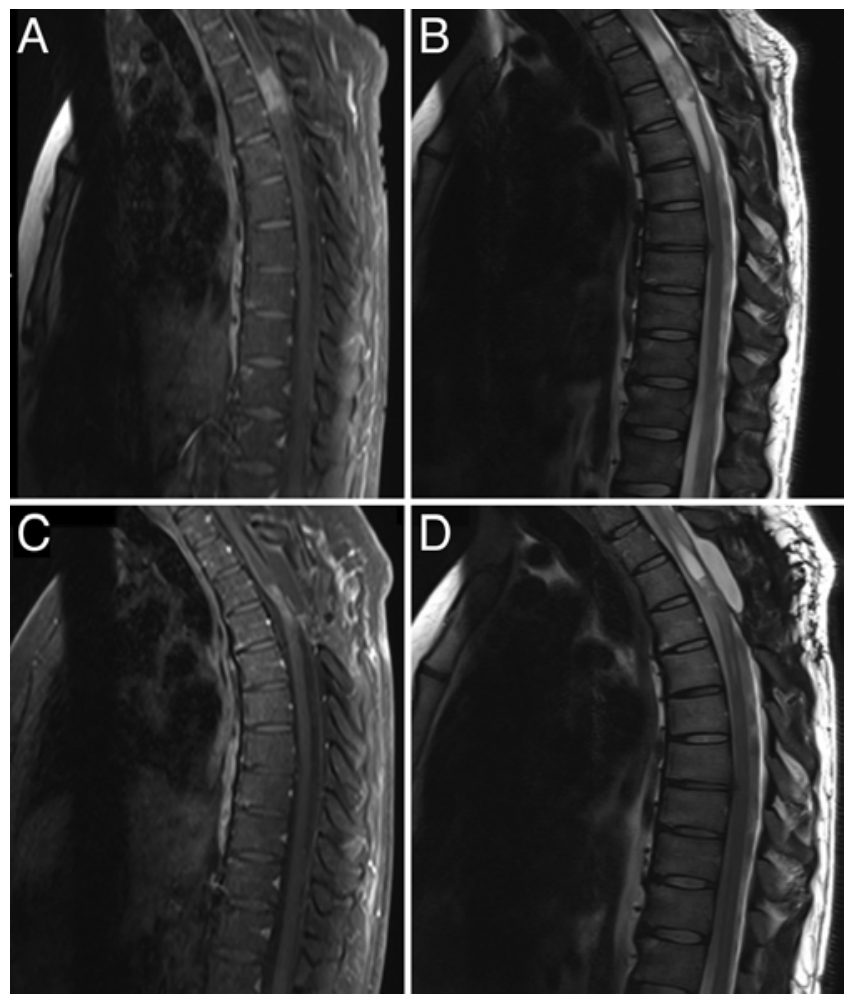

FIG. 2. Case 1. Pre- and postoperative representative MR images. A and $B$ : Preoperative sagittal T1-weighted image with contrast enhancement $(A)$ and sagittal T2-weighted image $(B)$ showing a contrastenhancing intramedullary tumor with associated cysts and peritumoral cord edema. C and D: Postoperative sagittal T1-weighted image with contrast enhancement $(C)$ and sagittal T2-weighted image (D) showing the resection cavity.
$50 \%$ concurrent with the tcMEP warnings. Along with the transient alarms, the CUSA activated the right abductor hallucis at a stimulation intensity of $0.5 \mathrm{~mA}$ (Fig. 3).

Postoperatively, there were no new motor deficits. The patient experienced loss of proprioception and diffuse hypesthesia, which gradually improved. Postoperative MRI demonstrated gross-total tumor resection (Fig. 2). The patient was discharged in good clinical condition, fully ambulating. A final pathology report revealed an ependymoma (WHO Grade II).

All 6 case-based clinical and neurophysiological experiences are summarized in Table 1.

\section{Discussion}

\section{IONM in IMSCT Surgery}

This is the first case-based study demonstrating a novel technique employing continuous intraoperative neurophysiological nerve mapping alongside classic monitoring in surgery for IMSCTs. Our current experience demonstrates the feasibility of using an ultrasonic aspirator connected to an electrode for continuous monitoring during IMSCT surgery. This fusion of technologies demonstrates that continuous motor evoked data could assist the surgeon, as it provides real-time detection of motor pathway proximity. The end goal of the introduction of this method would be to prevent permanent motor deficits based on a warning system that alerts the surgeon before iatrogenic injury is documented by classic monitoring.

\section{Mapping Versus Monitoring}

The application of neurophysiological recordings in neurosurgery has 2 main goals: mapping of "functional" neural elements and monitoring of eloquent pathways. Mapping is the process of delineating functional areas. This gives the surgeon a snapshot of the estimated danger zones of entry and allows for a tailored surgical plan. Monitoring is the process of continuously assessing functional neural integrity. Conceptually, during resection of an IMSCT, once the monitoring is disrupted, there is a high probability that the integrity of neural elements has already been compromised, either in a reversible or nonreversible manner. The current suggested protocols use multimodality IONM consisting of SSEP and musclebased tcMEP along with D-wave monitoring. ${ }^{34}$ Although this combination is widely accepted, there is still a search for an improved monitoring and mapping paradigm, as significant morbidity remains common in these complex and challenging surgeries. ${ }^{27,28,35}$ Historically, only SSEPs were monitored during spinal cord procedures. However, due to numerous reported false-negative results, ${ }^{16,18}$ i.e., postoperative motor deficit despite unchanged intraoperative SSEPs, use of SSEPs as a single modality has been largely abandoned. These supposed false-negative results are due to the fact that SSEPs do not reflect the functional integrity of motor pathways, and thus are not designed or expected to be sensitive to pure motor deficits. The role of monitoring sensory pathways in ISMCT surgery remains debatable, as attenuated SSEPs are common at myelotomy stages, even before lesion access and resection. Dorsal column mapping via recording of the ascending lemniscal 
A

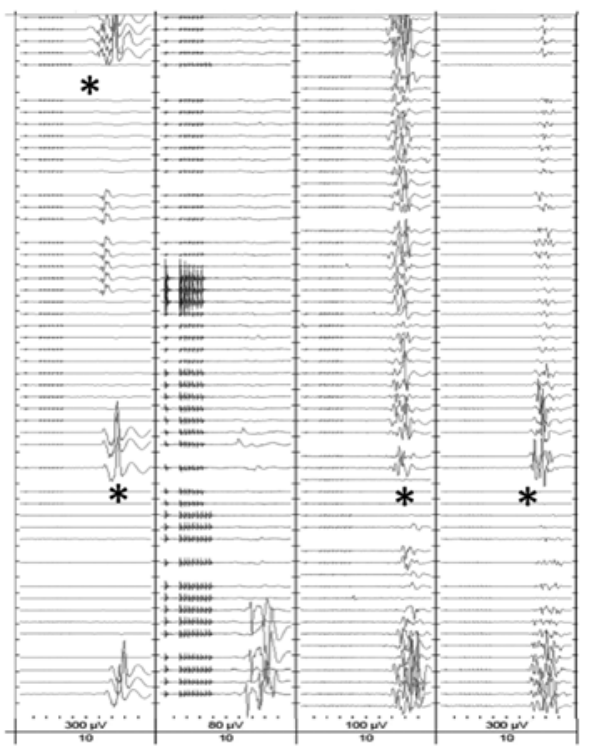

C

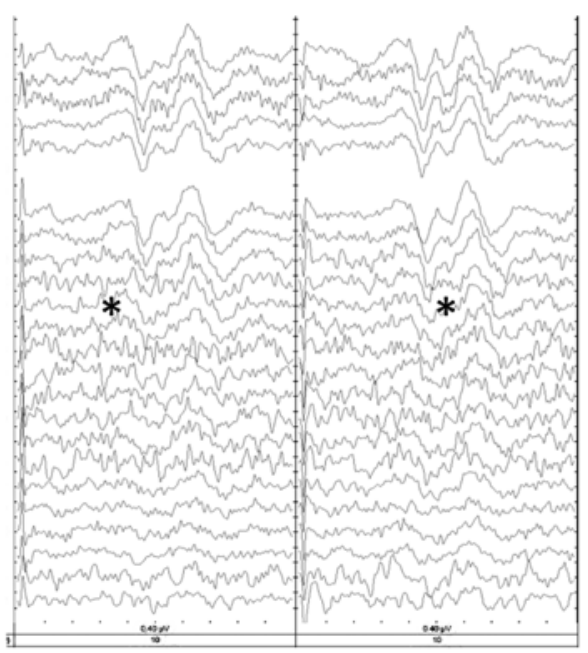

B

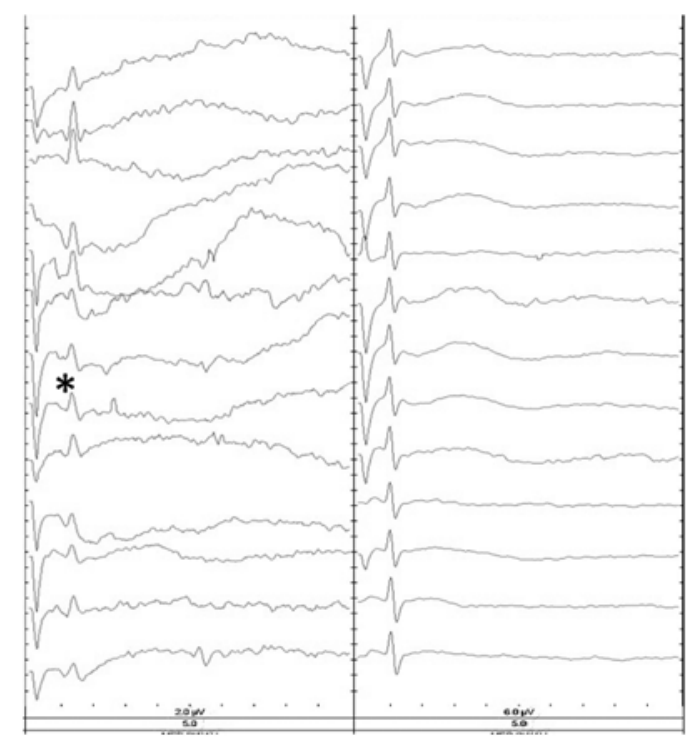

D

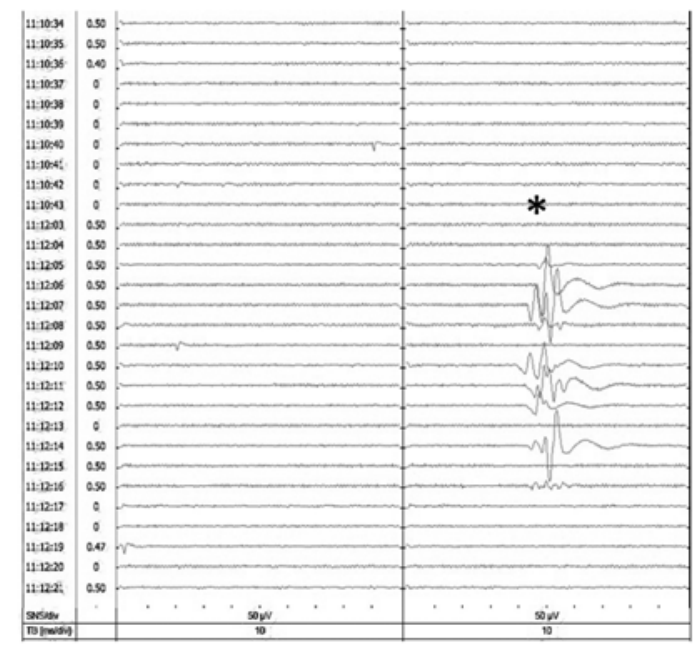

FIG. 3. Case 1. Neurophysiological data. A: TcMEP data (from right to left: left tibialis anterior, right tibialis anterior, left abductor hallucis, and right abductor hallucis muscles) showing lability of left- and right-sided data. B: D-wave data (caudal [left] and rostral [right] to the tumor) showing attenuation of caudal D-wave amplitude and stability of rostral amplitude. C: Abolishment of SSEP data at myelotomy (left [left] and right [right] posterior tibial nerve SSEPs [Cz-Fpz montage]). D: Recordings during mapping with CUSA showing activation of right abductor hallucis muscle with a stimulation intensity of $0.5 \mathrm{~mA}$ (vertical scale $50 \mu \mathrm{V}$ per division, horizontal scale $10 \mathrm{msec}$ ). Onset of significant attenuation is noted by the asterisks.

system conduction has been proposed to assist in identification of the anatomical and physiological midline for an optimally safe myelotomy entry point. ${ }^{23,29}$ Recently, Ando et al. demonstrated their experience with direct brain and spinal cord sensory stimulation in a method that resembles the D-wave technique but for sensory pathways. ${ }^{1}$

The combination of SSEPs and muscle-based tcMEPs has been shown to be of higher specificity and sensitivity than single modalities. ${ }^{17}$ The advantages of monitoring of motor pathways have been widely advocated previously. A statement by the American Society of Neurophysiological Monitoring ${ }^{25}$ demonstrates several examples of reversible muscle-based tcMEP deterioration with no new motor deficits, which provide circumstantial evidence for injury prevention..$^{23,31}$ These examples also provide consistent evidence that muscle-based tcMEP deterioration often occurs before and sometimes without SSEP changes, thus suggesting a greater chance for early detection, intervention, and motor-deficit prevention. A well-designed historical case-control study of IMSCT surgery demonstrated significantly better long-term outcomes with combined muscle-based tcMEP/D-wave monitoring compared with no monitoring. ${ }^{35}$ Additionally, a review of descending aortic surgery provided evidence for reduced paraplegia rates with muscle-based tcMEP monitoring compared with other monitoring techniques or unmonitored surgery. ${ }^{14}$

Although difficult to estimate, changes in muscle-based tcMEP morphology have been suggested to predict motor deficits. ${ }^{31}$ It is important to note that it is difficult to continuously monitor muscle-based tcMEP data due to patient 
movement resulting from transcranial stimulation, which is often paused during the most crucial stages of resection. Aside from altering the rhythm of surgery, these pauses for IONM testing may occur too late, possibly missing the stage of pyramidal injury leading to a permanent deficit. D-wave monitoring is considered an important addition to classic muscle-based tcMEP intraoperative alarms as it correlates with long-term motor function. Maintaining D-wave amplitude above $50 \%$ is considered indicative of intact neurological outcome in the long term. ${ }^{28,34} \mathrm{D}$-wave monitoring is critical in these surgeries as its data are both quantitative and continuous throughout surgery. D-wave information can be nondiscriminating to unilateral injury and is not possible when lesions are at or below the T-10 spinal level. ${ }^{13}$ Aside from muscle-based tcMEP and D-waves, free-running electromyography has also been suggested as a method to detect early motor-tract injury during IMSCT surgery. ${ }^{38}$

IONM is considered by many to be a deserved standard in all surgeries for IMSCT. Nevertheless, despite supportive data, there is still no Level 1 proof for the efficacy of IONM in preventing injury during surgery for intramedullary tumors. ${ }^{24,28,34}$

\section{Dynamic Mapping of the CST}

Mapping of the spinal cord motor elements has gained little attention in the literature. Conversely, mapping of the CST fibers in supratentorial surgery has gathered much attention over the past several years ${ }^{13,40}$ and has been shown to be accurate and effective in quantitatively identifying proximity to the CSTs. ${ }^{30}$ It has also been shown that subcortical mapping reduces morbidity in the supratentorial patient group. ${ }^{2}$ Recently, Shiban et al. demonstrated the feasibility of continuous motor mapping using subcortical stimulation via a surgical aspirator in cranial surgery. In comparison with the sequential use of a standard monopolar stimulation probe, this was found to be a feasible and safe method with few disadvantages. ${ }^{37}$ Compared with the standard probe, the aspirator offers continuous information on the excitability of the tracts, and therefore continuously estimates the distance of the probe tip to the CST. Previously, in a similar concept, Raabe et al. described mapping of the CST using the tip of a suction device along with amplified acoustic display, suggesting that these continuous adjuncts lead to increased safety during resection. ${ }^{32}$

\section{Validity of Technique}

The technical nuances and distance from motor tracts with respect to stimulation thresholds remain controversial even in supratentorial surgery. ${ }^{36}$ Nossek et al. used a combination of neurophysiological assessment, tractographybased neuronavigation, and updated intraoperative ultrasound. This combination provided accurate localization of the CSTs and demonstrated a linear correlation between the distance to the CSTs and the threshold of subcortical stimulation, suggesting a 1-mA:1-mm threshold intensity to distance ratio correlation. ${ }^{30}$ The correlation between the stimulation thresholds and the distance from motor tracts in the spinal cord has yet to be determined. Establishing neurophysiological and image-based correlations within the cord will be challenging due to the lack of standard imaging protocols as well as the smaller scale and tighter fiber bundling compared with the supratentorial context.

Gandhi et al. described the use of a bipolar probe during resection of a cervicomedullary junction cystic ependymoma to perform high-resolution microstimulation motor mapping of CSTs that are responsible for individual muscle groups. ${ }^{15}$ This technique was not based on the aspirator, but, similar to data presented in our current study, aims at mapping the motor fibers during resection and minimizing the risk of surgical morbidity.

Our technique involves nonspecific stimulation of the spinal cord and its sub-elements. And while it is scientifically sound to assume that stimulation of the CSTs within the cord will result in an evoked motor response (i.e., a sensitive positive mapping result), we cannot rule out that stimulation of other fibers within the spinal cord, such as propriospinal fibers supporting corticospinal conduction or those involved in centrally mediated reflexes, will similarly result in a recorded motor response. Due to the expectation of similar latencies of muscle responses generated either by true CST activation or propriospinal fiber or reflex fiber stimulation, it would be difficult to differentiate between these scenarios on the basis of muscle response onset alone. In our current experience, for each instance of motor evoked activity by the CUSA, the motor response distribution was appropriate for the spinal level and side of the cord that was being stimulated.

Deletis and Bueno De Camargo ${ }^{12}$ described a technique to map the CST in intramedullary surgery using a D-wave collision technique. They described simultaneous stimulation of the spinal cord with a monopolar stimulator at 2 $\mathrm{mA}$ together with transcranial stimulation used to evoke the classic D-wave. Since each stimulation site activates the CST independently, the descending and ascending volleys in the identical fibers at least partially cancel each other out when the location of the spinal cord stimulator is within $1 \mathrm{~mm}$ of either CST. This technique has the advantage of specific mapping since the D-wave is specifically CST mediated, to the exclusion of other spinal cord circuitry. However, the collision technique may be challenging on a practical level, due to concerns of laterality, signal interpretation, dynamic sensitivity, and nonapplicability in lesions below T-10. However, future studies comparing both techniques would serve as a good validation of cord stimulation-based CST mapping.

\section{Technical Considerations}

From our experience early in the series, some modification of the classic subcortical mapping parameters was necessary. Stimulation under classic parameters (cathodal trains-of-five pulses with an interstimulus interval of 3-4 msec, 500- $\mu$ sec duration per pulse) yielded responses in an oversensitive manner, giving warning at areas of the tumor that were visually distant to the pyramidal tracts. Another reason for alteration was the masking of responses by the relatively long stimulus artifact. Using classic trainof-five pulses at the accepted supratentorial parameters, ${ }^{30}$ a response faster than 14.5-18.5 msec would be obscured. In supratentorial or spinal surgery, where the location of CST activation is far from the muscle target, this is not problematic, but in the context of IMSCTs it is of concern. 
Therefore, we modified the technique to use train-of-three pulses and at $200 \mu \mathrm{sec}$, each with an interstimulus interval of $3 \mathrm{msec}$. This limited the stimulus artifact to an ending latency of $6.2 \mathrm{msec}$, supporting the recording of earlylatency motor responses.

We appreciate that there are various techniques for removing IMSCTs, and using a handheld suction device is also common. The ultrasonic aspirator settings can be adjusted during resection of tumor margins to offer suction only while still allowing for continuous mapping. Thus, in our opinion, this technique is generalizable for various surgical preferences.

\section{Limitations}

Certain limitations must be considered when using this multipurpose aspirator. The room for error in surgery for IMSCTs is minimal, and the optimal stimulation settings have yet to be determined. Undersensitive stimulation may lead to irreversible neural damage, while oversensitivity of the stimulation may lead to false-positive alarms, ending with suboptimal resections and leading to a suboptimal oncological result. Our current series is too small to accurately estimate the rates of specificity and sensitivity, neither for false-positive nor for false-negative alarms, thus making solid conclusions impossible at this point.

Another limitation is the dynamics of monitoring versus CUSA mapping. Since it is technically impossible to perform both simultaneously, the recording of one is at the expense of the other. In our study we alternated use of both, but ultimately we used the classic monitoring signs as indications for surgical intervention and postoperative prognostic evaluation. Future studies will need to delineate the hierarchal relationship between mapping and monitoring methodologies, as has been described in supratentorial surgery. ${ }^{36}$

The ultrasonic aspirator itself may affect the IONM data when used on the dura or the spinal cord, ${ }^{39}$ altering potentials in a manner unrelated to the resection. Furthermore, the relationship between the ultrasonic aspirator and monitoring of corticospinal pathways can potentially be misleading, as it has been shown that the use of a Cavitron aspirator in proximity to motor pathways may temporarily inhibit motor conduction in cortical mapping ${ }^{5}$ by a facilitatory effect due to the combination of both the electrical and mechanical stimulation.

However, in our experience using this methodology in supratentorial surgery, we have not found either to be an obvious confounding concern. Future studies will need to be designed to delineate the differences in excitability between cranial-based and cord-based CST excitation.

\section{Conclusions}

Continuous monitoring of motor tracts during IMSCT resection using a multipurpose resective tool is feasible and safe. It provides real-time continuous data regarding the integrity of motor functions and may serve as an alarm to proximity to the motor tracts prior to physical compromise, diminishing surgical risk in these complex resections. Although it remains to be validated, this new surgical adjunct has groundbreaking potential in resection of IMSCTs. The efficacy of this method needs to be determined in larger scale studies. Many technical variables need to be considered, including the correlation to $\mathrm{D}$-wave and muscle tcMEP monitoring. The importance of the continuous dialog between the surgical and monitoring teams cannot be overemphasized. The novel technique described herein appears to be promising, safe, and feasible. Larger series will determine whether it is beneficial.

\section{Acknowledgments}

We thank Mrs. Adina Sherer, MSc, for her assistance in editing this manuscript.

\section{References}

1. Ando M, Tamaki T, Yoshida M, Kawakami M, Kubota S, Nakagawa Y, et al: Intraoperative spinal cord monitoring using combined motor and sensory evoked potentials recorded from the spinal cord during surgery for intramedullary spinal cord tumor. Clin Neurol Neurosurg 133:18-23, 2015

2. Bello L, Riva M, Fava E, Ferpozzi V, Castellano A, Raneri F, et al: Tailoring neurophysiological strategies with clinical context enhances resection and safety and expands indications in gliomas involving motor pathways. Neuro Oncol 16:1110-1128, 2014

3. Berhouma M, Bahri K, Houissa S, Zemmel I, Khouja N, Aouidj L, et al: [Management of intramedullary spinal cord tumors: surgical considerations and results in 45 cases.] Neurochirurgie 55:293-302, 2009 (Fr)

4. Boström A, Kanther NC, Grote A, Boström J: Management and outcome in adult intramedullary spinal cord tumours: a 20-year single institution experience. BMC Res Notes 7:908, 2014

5. Carrabba G, Mandonnet E, Fava E, Capelle L, Gaini SM, Duffau H, et al: Transient inhibition of motor function induced by the Cavitron ultrasonic surgical aspirator during brain mapping. Neurosurgery 63:E178-E179, 2008

6. Cheng JS, Ivan ME, Stapleton CJ, Quinones-Hinojosa A, Gupta N, Auguste KI: Intraoperative changes in transcranial motor evoked potentials and somatosensory evoked potentials predicting outcome in children with intramedullary spinal cord tumors. J Neurosurg Pediatr 13:591-599, 2014

7. Constantini S, Epstein F: Surgical management of intramedullary spinal cord tumors, in Schmidek, Sweet (eds): Operative Neurosurgical Techniques, ed 3. Philadelphia: Saunders, 1995, pp 2095-2106

8. Constantini S, Epstein F: Ultrasonic dissection, in Wilkins RH, Rengachary SS (eds): Neurosurgery, ed 2. New York: McGraw-Hill, 1996, pp 607-608

9. Constantini S, Houten J, Miller DC, Freed D, Ozek MM, Rorke LB, et al: Intramedullary spinal cord tumors in children under the age of 3 years. J Neurosurg 85:1036-1043, 1996

10. Constantini S, Miller DC, Allen JC, Rorke LB, Freed D, Epstein FJ: Radical excision of intramedullary spinal cord tumors: surgical morbidity and long-term follow-up evaluation in 164 children and young adults. J Neurosurg 93 (2 Suppl):183-193, 2000

11. Costa P, Peretta P, Faccani G: Relevance of intraoperative D wave in spine and spinal cord surgeries. Eur Spine J 22:840-848, 2013

12. Deletis V, Bueno De Camargo A: Interventional neurophysiological mapping during spinal cord procedures. Stereotact Funct Neurosurg 77:25-28, 2001

13. Deletis V, Sala F: Subcortical stimulation (mapping) of the corticospinal tract. Clin Neurophysiol 122:1275-1276, 2011

14. Dong CC, MacDonald DB, Janusz MT: Intraoperative spinal cord monitoring during descending thoracic and thoracoab- 
dominal aneurysm surgery. Ann Thorac Surg 74:S1873S1876, S1892-S1878, 2002

15. Gandhi R, Curtis CM, Cohen-Gadol AA: High-resolution direct microstimulation mapping of spinal cord motor pathways during resection of an intramedullary tumor. J Neurosurg Spine 22:205-210, 2015

16. Ginsburg HH, Shetter AG, Raudzens PA: Postoperative paraplegia with preserved intraoperative somatosensory evoked potentials. Case report. J Neurosurg 63:296-300, 1985

17. Hyun SJ, Rhim SC: Combined motor and somatosensory evoked potential monitoring for intramedullary spinal cord tumor surgery: correlation of clinical and neurophysiological data in 17 consecutive procedures. Br J Neurosurg 23:393400, 2009

18. Jones SJ, Buonamassa S, Crockard HA: Two cases of quadriparesis following anterior cervical discectomy, with normal perioperative somatosensory evoked potentials. J Neurol Neurosurg Psychiatry 74:273-276, 2003

19. Juthani RG, Bilsky MH, Vogelbaum MA: Current management and treatment modalities for intramedullary spinal cord tumors. Curr Treat Options Oncol 16:39, 2015

20. Karikari IO, Nimjee SM, Hodges TR, Cutrell E, Hughes BD, Powers CJ, et al: Impact of tumor histology on resectability and neurological outcome in primary intramedullary spinal cord tumors: a single-center experience with 102 patients. Neurosurgery 76 (Suppl 1):S4-S13, 2015

21. Klekamp J: Treatment of intramedullary tumors: analysis of surgical morbidity and long-term results. J Neurosurg Spine 19:12-26, 2013

22. Korn A, Halevi D, Lidar Z, Biron T, Ekstein P, Constantini S: Intraoperative neurophysiological monitoring during resection of intradural extramedullary spinal cord tumors: experience with 100 cases. Acta Neurochir (Wien) 157:819-830, 2015

23. Kothbauer KF, Deletis V, Epstein FJ: Motor-evoked potential monitoring for intramedullary spinal cord tumor surgery: correlation of clinical and neurophysiological data in a series of 100 consecutive procedures. Neurosurg Focus 4(5):e1, 1998

24. Lall RR, Lall RR, Hauptman JS, Munoz C, Cybulski GR, Koski T, et al: Intraoperative neurophysiological monitoring in spine surgery: indications, efficacy, and role of the preoperative checklist. Neurosurg Focus 33(5):E10, 2012

25. Macdonald DB, Skinner S, Shils J, Yingling C: Intraoperative motor evoked potential monitoring - a position statement by the American Society of Neurophysiological Monitoring. Clin Neurophysiol 124:2291-2316, 2013

26. Manzano G, Green BA, Vanni S, Levi AD: Contemporary management of adult intramedullary spinal tumors-pathology and neurological outcomes related to surgical resection. Spinal Cord 46:540-546, 2008

27. McGirt MJ, Constantini S, Jallo GI: Correlation of a preoperative grading scale with progressive spinal deformity following surgery for intramedullary spinal cord tumors in children. J Neurosurg Pediatr 2:277-281, 2008

28. Morota N, Deletis V, Constantini S, Kofler M, Cohen H, Epstein FJ: The role of motor evoked potentials during surgery for intramedullary spinal cord tumors. Neurosurgery 41:1327-1336, 1997

29. Nair D, Kumaraswamy VM, Braver D, Kilbride RD, Borges LF, Simon MV: Dorsal column mapping via phase reversal method: the refined technique and clinical applications. Neurosurgery 74:437-446, 2014

30. Nossek E, Korn A, Shahar T, Kanner AA, Yaffe H, Marcovici $\mathrm{D}$, et al: Intraoperative mapping and monitoring of the corticospinal tracts with neurophysiological assessment and 3-dimensional ultrasonography-based navigation. Clinical article. J Neurosurg 114:738-746, 2011

31. Quiñones-Hinojosa A, Lyon R, Zada G, Lamborn KR, Gupta
$\mathrm{N}$, Parsa AT, et al: Changes in transcranial motor evoked potentials during intramedullary spinal cord tumor resection correlate with postoperative motor function. Neurosurgery 56:982-993, 2005

32. Raabe A, Beck J, Schucht P, Seidel K: Continuous dynamic mapping of the corticospinal tract during surgery of motor eloquent brain tumors: evaluation of a new method. J Neurosurg 120:1015-1024, 2014

33. Raco A, Esposito V, Lenzi J, Piccirilli M, Delfini R, Cantore G: Long-term follow-up of intramedullary spinal cord tumors: a series of 202 cases. Neurosurgery 56:972-981, 2005

34. Sala F, Bricolo A, Faccioli F, Lanteri P, Gerosa M: Surgery for intramedullary spinal cord tumors: the role of intraoperative (neurophysiological) monitoring. Eur Spine J 16 (Suppl 2):S130-S139, 2007

35. Sala F, Palandri G, Basso E, Lanteri P, Deletis V, Faccioli F, et al: Motor evoked potential monitoring improves outcome after surgery for intramedullary spinal cord tumors: a historical control study. Neurosurgery 58:1129-1143, 2006

36. Seidel K, Beck J, Stieglitz L, Schucht P, Raabe A: The warning-sign hierarchy between quantitative subcortical motor mapping and continuous motor evoked potential monitoring during resection of supratentorial brain tumors. J Neurosurg 118:287-296, 2013

37. Shiban E, Krieg SM, Obermueller T, Wostrack M, Meyer B, Ringel F: Continuous subcortical motor evoked potential stimulation using the tip of an ultrasonic aspirator for the resection of motor eloquent lesions. J Neurosurg 123:301-306, 2015

38. Skinner SA, Nagib M, Bergman TA, Maxwell RE, Msangi G: The initial use of free-running electromyography to detect early motor tract injury during resection of intramedullary spinal cord lesions. Neurosurgery 56 (2 Suppl):299-314, 2005

39. Suetsuna F, Harata S, Yoshimura N: Influence of the ultrasonic surgical aspirator on the dura and spinal cord. An electrohistologic study. Spine (Phila Pa 1976) 16:503-509, 1991

40. Szelényi A, Senft C, Jardan M, Forster MT, Franz K, Seifert $\mathrm{V}$, et al: Intra-operative subcortical electrical stimulation: a comparison of two methods. Clin Neurophysiol 122:14701475, 2011

41. Tobias ME, McGirt MJ, Chaichana KL, Goldstein IM, Kothbauer KF, Epstein F, et al: Surgical management of long intramedullary spinal cord tumors. Childs Nerv Syst 24:219-223, 2008

\section{Disclosures}

The authors report no conflict of interest concerning the materials or methods used in this study or the findings specified in this paper.

\section{Author Contributions}

Conception and design: Constantini, Barzilai, Korn. Acquisition of data: all authors. Analysis and interpretation of data: Constantini, Barzilai, Bitan-Talmor, Korn. Drafting the article: Barzilai, Korn. Critically revising the article: Constantini, Barzilai, Salame, Korn. Reviewed submitted version of manuscript: Constantini, Barzilai, Lidar, Salame, Korn. Approved the final version of the manuscript on behalf of all authors: Constantini. Administrative/ technical/material support: Barzilai, Bitan-Talmor. Study supervision: Constantini, Barzilai, Lidar, Salame, Korn.

\section{Correspondence}

Shlomi Constantini, Department of Pediatric Neurosurgery, The Israeli Neurofibromatosis Center, Dana Children's Hospital, Tel Aviv Medical Center, Weizman 6, Tel Aviv, Israel 64239. email: sconsts@netvision.net.il. 